\title{
Stress regulation and cognitive control: evidence relating cortisol reactivity and neural responses to errors
}

\author{
Rebecca J. Compton • Julia Hofheimer • \\ Rebecca Kazinka
}

Published online: 10 October 2012

(C) Psychonomic Society, Inc. 2012

\begin{abstract}
In this study, we tested the relationship between error-related signals of cognitive control and cortisol reactivity, investigating the hypothesis of common systems for cognitive and emotional self-regulation. Eighty-three participants completed a Stroop task while electroencephalography (EEG) was recorded. Three error-related indices were derived from the EEG: the error-related negativity (ERN), error positivity $(\mathrm{Pe})$, and error-related alpha suppression (ERAS). Pre- and posttask salivary samples were assayed for cortisol, and cortisol change scores were correlated with the EEG variables. Better error-correct differentiation in the ERN predicted less cortisol increase during the task, whereas greater ERAS predicted greater cortisol increase during the task; the Pe was not correlated with cortisol changes. We concluded that an enhanced ERN, part of an adaptive cognitive control system, predicts successful stress regulation. In contrast, an enhanced ERAS response may reflect errorrelated arousal that is not adaptive. The results support the concept of overlapping systems for cognitive and emotional self-regulation.
\end{abstract}

Keywords Cognitive control · Emotion · Event-related potentials

In recent years, researchers across various subdisciplines within psychology have been concerned with understanding self-regulation: namely, how behavioral output is controlled and adjusted by higher-level systems in order to adequately meet goals (e.g., Baumeister \& Vohs, 2004). The interest in self-regulation has emerged in different subfields that sometimes use different terminology, but that in a general sense

\footnotetext{
R. J. Compton $(\varangle) \cdot J$. Hofheimer $\cdot$ R. Kazinka Department of Psychology, Haverford College, 370 Lancaster Avenue, Haverford, PA 19041, USA

e-mail: rcompton@haverford.edu
}

are focused on adaptive behavior change (Hofmann, Schmeichel, \& Baddeley, 2012; MacKenzie, Mezo, \& Francis, 2012). For example, within the subfield of cognitive neuroscience, researchers have studied processes of "cognitive control," through which the mind responds to its own errors and conflicts by enhancing attention to taskrelevant events and attributes (e.g., Botvinick, Braver, Barch, Carter, \& Cohen, 2001). In the realm of affect, researchers have studied emotion regulation, through which the mind controls the experience and expression of emotion (e.g., Gross, 2007).

A central theoretical question is the extent to which selfregulation across different domains draws upon the same general-purpose processes. MacKenzie et al. (2012) argued that all models of self-regulation include several components: a representation of the goal, a perception of the current state, a process of comparison between those two, and a response process that corrects discrepancies between the goal and the present state. Current cognitive neuroscience work has focused on specific regions of the frontal lobe, such as the anterior cingulate, dorsolateral prefrontal cortex, and right inferior frontal gyrus, as crucial for detection of goal-state discrepancies and for implementation of corrective control (e.g., Ridderinkhof, van den Wildenberg, Segalowitz, \& Carter, 2004; Tops \& Boksem, 2011b; van Veen \& Carter, 2006). These regions are implicated in studies of both the control of cognitive task performance and the control of emotions (e.g., Ochsner \& Gross, 2005; Zelazo \& Cunningham, 2007), implicating a "common-systems" view of self-regulation across both cognitive and emotional domains.

Some researchers have addressed the relationship between cognitive control and emotion regulation through a theoretical framework focused on mental resources. If general-purpose control systems exist and have limited capacity, then depleting those resources for one control function ought to have consequences for another. For example, engaging in a challenging task of executive control had negative consequences for subsequent emotion regulation 
(Schmeichel, 2007). Conversely, manipulating emotion regulation experiences has consequences for error-related cognitive control processes (Inzlicht \& Gutsell, 2007). The particular measurement of cognitive control that researchers have focused on, one that is relevant to the present study, is the error-related negativity (ERN). The ERN is an eventrelated potential derived from scalp electroencephalography (EEG) recordings that occurs within $100 \mathrm{~ms}$ of an erroneous buttonpress (Falkenstein, Hohnsbein, Hoormann, \& Blanke, 1991; Gehring, Goss, Coles, Meyer, \& Donchin, 1993; for reviews, see Gehring, Liu, Orr, \& Carp, 2012; Simons, 2010). The ERN is thought to reflect an error-detection process within the anterior cingulate cortex, presumed to be part of a cognitive control system that monitors for outcomes that are worse than expected (Holroyd \& Coles, 2002). Crucially, Inzlicht and Gutsell found that ERN amplitudes were dampened after participants were instructed to suppress negative emotions elicited by a film. These results imply a common pool of resources that can be tapped by both emotion regulation and error-related cognitive control tasks.

Research on individual differences has also supported the close relationship between error-related cognitive control and emotion regulation. In two studies, individual differences in neural and behavioral responses to performance mistakes predicted daily reports of mood and coping (Compton, Arnstein, Freedman, Dainer-Best, Liss, \& Robinson, 2011; Compton et al., 2008). Specifically, participants with effective error detection (measured by the ERN) and adaptive posterror behavior change tended to show less self-reported negative affect and more task-focused coping behaviors in response to daily stressors reported over a two-week period. These results imply that people who engage in effective error detection during a cognitive task in the laboratory also tend to engage in effective stress regulation in everyday life. However, one limitation of this prior work is that it relied on self-report measures of stress reactivity, which have strong face validity but are not the only means of assessing reactivity to stress.

To seek converging evidence for the relationship between error-related cognitive control and emotion regulation from an individual-differences perspective, in the present study we examined cortisol reactivity. Cortisol, a steroid hormone released as part of the body's response to stressors, can be measured reliably in saliva samples and is responsive to psychological stressors in laboratory settings (see Dickerson \& Kemeny, 2004, for review). Anatomical evidence has provided a plausible neural path for cognitive control structures to influence cortisol regulation. In particular, descending projections from medial prefrontal cortex (MPFC) regions make direct contact with the hypothalamus, as well as with the amygdala, which can influence the hypothalamus (Barbas,
Saha, Rempel-Clower, \& Ghashghaei, 2003; for a review, see Ray \& Zald, 2012). The hypothalamus, in turn, influences cortisol release through the hypothalamus-pituitary-adrenal axis. Although it is unknown exactly what functions are served by the MPFC-hypothalamic path, it is at least plausible that cognitive control of the stress response could be one of those functions. Conversely, glucocorticoids can also impact the functioning of the MPFC and its interaction with the amygdala (e.g., Barsegyan, McKenzie, Kurose, McGaugh, \& Roozendaal, 2010; Roozendaal et al., 2009; Veer et al., 2012).

Prior studies have provided preliminary, albeit mixed, evidence associating cortisol responsiveness with errorrelated cognitive control. Tops, Boksem, Wester, Lorist, and Meijman (2006) reported that higher ERN amplitudes were associated with a greater decrease in cortisol during a task session, albeit in a relatively small sample of 18 participants. Cavanagh and Allen (2008) also found that ERN amplitudes were associated with cortisol activity, but in opposite directions for participants who were low and high in behavioral inhibition tendencies. Finally, in a preliminary study, Amen, Dodd, Fineman, and Compton (2008) reported that ERN amplitudes predicted reduced cortisol increases during a Stroop task, but outliers may have exerted undue influence on their results. Given the equivocal evidence from these prior studies, in the present study we sought to determine, in a relatively large sample, whether greater ERN amplitudes are associated with enhanced regulation of cortisol during a cognitive task.

In addition to studying the relationship between cortisol regulation and the ERN, in the present study we also examined cortisol's relationship with two other error-related neural signals derived from EEG recordings, namely the error positivity $(\mathrm{Pe})$ and error-related alpha suppression (ERAS). These two neural markers reliably follow errors in performance but have been studied less than the ERN. The Pe is an event-related potential that occurs about $200-400 \mathrm{~ms}$ after a response, is more positive-going on error trials than on correct trials, and typically has a more posterior scalp distribution than the ERN (Overbeek, Nieuwenhuis, \& Ridderinkhof, 2005). Its functional significance vis-à-vis the ERN is not completely clear, but two alternative possibilities are (1) that, as compared to the ERN, it is more closely tied to autonomic reactions to errors (Hajcak, McDonald, \& Simons, 2003) and (2) that it reflects a cognitive P3 "oddball" response to rare events, namely errors that occur infrequently amongst correct responses (Arbel \& Donchin, 2009; Ridderinkhof, Ramautar, \& Wijnen, 2009). If the first of these two alternatives is correct, the Pe amplitude may covary with cortisol regulation to an even greater extent than the ERN.

The third error-related neural marker, ERAS, differs from the ERN and Pe in that it is derived from quantifying oscillatory 
activity in the EEG rather than from time-locked event-related potentials. In prior work, we demonstrated an increase in EEG alpha power during the intertrial interval following correct responses, as compared to a relatively suppressed pattern of alpha activity following error responses (Carp \& Compton, 2009; Compton, Arnstein, Freedman, Dainer-Best, \& Liss, 2011). Because alpha activity is inversely correlated with mental arousal, we infer that the ERAS pattern reflects relatively greater arousal in the intertrial interval following errors than on correct trials. Our prior findings also suggested that ERAS may be functionally separable from the ERN, in that ERN amplitudes predicted posterror accuracy, whereas ERAS effects predicted posterror slowing (Carp \& Compton, 2009). As with the Pe, the functional significance of ERAS is still poorly understood, but one possibility is that it reflects an arousal component of reaction to errors, rather than an adaptive control component (Carp \& Compton, 2009). If this latter hypothesis is true, we might expect participants with greater ERAS effects to show increased levels of cortisol during the cognitive task, contrary to the prediction involving the ERN.

\section{Method}

\section{Participants}

Data were drawn from participants in two separate samples who completed very similar cognitive tasks (described in the next section). The samples were combined to increase statistical power. As part of a larger study with a different purpose, both original samples included participants preselected for either low or high levels of depression. For the present purposes, only data from the 87 nondepressed participants were considered (Sample 1 consisted of 16 males and 34 females with Beck Depression Inventory scores between 0 and 9; Sample 2 consisted of 19 males and 18 females with Center for Epidemiological Studies Depression Scale scores between 0 and 9.5). Some data from the participants in Sample 1 have previously been reported (Compton, Arnstein, Freedman, Dainer-Best, Liss, \& Robinson, 2011), but that prior report did not include cortisol data, which is the focus of the present study. All of the participants were undergraduates who self-reported no prior history of neurological deficits, no current use of substances that affect the nervous system (e.g., antidepressants or anxiolytics), and normal vision. Four of the participants were later excluded from the data analysis because of task accuracy below $60 \%(n=2)$ or lack of measurable cortisol in the saliva sample $(n=2)$. Therefore, the final data analyses were carried out on 83 participants ( 34 males and 49 females).

\section{Procedure overview}

After hearing an explanation of the task session and completing a consent form, the participants contributed their first saliva sample. Experimenters next applied the EEG cap and situated the participant in the testing room, and the participant then completed a Stroop task for approximately $30 \mathrm{~min}$. Immediately after the Stroop task, the participant contributed the second saliva sample. Although the total time between the first and second saliva samples differed somewhat across participants, due to vagaries in cap application and differences in the time taken for rests between blocks, generally the second sample was taken approximately $50 \mathrm{~min}$ after the first sample and 30 min after the beginning of the Stroop task. A meta-analysis by Dickerson and Kemeny (2004) indicated that effect sizes for cortisol reactivity peak 20-30 min after stressor onset.

\section{Task}

Participants completed a six-choice Stroop task that was similar for Samples 1 and 2. The task required the participants to identify the color (red, orange, yellow, green, blue, or purple) of a target word whose meaning was colorincongruent (i.e., color word in conflict with the font color; e.g., "red" in blue font), neutral (e.g., "chair"), or emotional (e.g., "fail"). Responses were indicated using the first three fingers of each hand on a computer keyboard.

The events on each trial included a 150 -ms presentation of the target word against a black background, following by a blank screen displayed until the participant responded. This was followed by a $1,280-\mathrm{ms}$ intertrial interval (ITI) with a blank screen on display, before the onset of the next trial. Participants completed 24 practice trials with accuracy feedback to ensure that they understood the stimulus-response mapping, but no feedback was given during the main experimental trials. Break screens between trial blocks reminded participants of the correct stimulus-response mapping.

The only difference between the tasks for Samples 1 and 2 was whether word types were blocked or intermixed. For Sample 1, word types were blocked, such that the task included seven color-word blocks, involving 30 colorincongruent and 30 neutral trials, and seven emotion-word blocks, involving 30 emotion and 30 neutral trials. The trials within a block were randomly intermixed, block order was randomized, and the total number of trials was 840 . For Sample 2, the task included ten blocks with all three word types intermixed within blocks. That is, 30 incongruent, 30 neutral, and 30 emotion trials were randomly intermixed within each of the ten blocks, for a total of 900 trials. As is described in more detail below, overall the responses were slower for participants in Sample 1 than in Sample 2, but 
otherwise all variables of interest in the present report were indistinguishable between the two samples. For the present report, we examined performance and EEG/ERP responses to errors versus correct trials, regardless of word types (incongruent, neutral, or emotional) for simplicity and because of the somewhat different task structures between the two samples.

\section{Electrophysiological recording and data processing}

Electrodes were applied using an elastic cap (Quik-Caps) fitted with sintered $\mathrm{Ag} / \mathrm{AgCl}$ electrodes. Data were recorded continuously from four midline scalp sites $(\mathrm{Fz}, \mathrm{FCz}, \mathrm{Cz}$, and $\mathrm{Pz}$ ) and three pairs of lateral sites (F3/4, C3/4, and P3/4). Signals were amplified by a NuAmps amplifier controlled by Neuroscan software, with a sampling rate of $1000 \mathrm{~Hz}$ and a bandpass of $0.1-40 \mathrm{~Hz}(-3 \mathrm{~dB})$. The data were referenced online to the left mastoid and digitally re-referenced offline to the average of the left and right mastoids. Eye movements were monitored by electrodes placed above and below the left eye and at the outer canthus of each eye. Recordings from these four sites were used to compute bipolar horizontal and vertical electrooculogram channels offline.

Artifacts were addressed offline in three steps. First, upon visual inspection, portions of the EEG record with large nonblink artifacts were manually excluded. Second, the effect of blinks was reduced using the Neuroscan software's regressionbased algorithm for ocular artifact reduction. Finally, remaining artifacts in the EEG were identified using a $\pm 150-\mu \mathrm{V}$ threshold, and the corresponding epochs were excluded.

Data processing to extract the ERN and Pe included creating epochs around each response marker (buttonpress onset), beginning $200 \mathrm{~ms}$ before and extending until $600 \mathrm{~ms}$ after the response. Epochs were baseline-corrected, with the baseline defined by voltages in the interval from 200 to $100 \mathrm{~ms}$ preresponse and averaged separately for correct and error trials. The ERN was defined as the peak negative amplitude between 0 and $100 \mathrm{~ms}$ postresponse, and the Pe was defined as the mean amplitude between 200 and $600 \mathrm{~ms}$ postresponse. Analyses of the ERN and Pe data focused only on the midline leads $(\mathrm{Fz}, \mathrm{FCz}, \mathrm{Cz}$, and $\mathrm{Pz})$ where these phenomena are typically observed (Gehring et al., 2012).

To address alpha power changes following errors versus correct trials, power spectra were computed for five 256-ms epochs beginning at the time of response and extending throughout the intertrial interval. Power spectra were obtained for each window using the fast Fourier transform and a cosine windowing method. This procedure yielded time frequency representations of the intertrial interval with a resolution of $256 \mathrm{~ms}$ in the time domain and $4 \mathrm{~Hz}$ in the frequency domain. Spectra for each window were then averaged separately for trial types (i.e., error vs. correct). The statistical analyses were conducted using log-transformed mean power values in the 10- to $14-\mathrm{Hz}$ frequency band.
Saliva sample and assay

Saliva samples were collected immediately before and after the Stroop task. Collection was by means of passive drool through a straw into micropipette tubes, which were then frozen and stored for later assay. The samples were sent to Salimetrics, LLC (State College, PA) for enzyme immunoassay in duplicate. The duplicate values were highly correlated $(r=0.993)$, confirming the reliability of the assay. Analyses were conducted on the mean of the duplicate values.

\section{Results}

\section{Cortisol}

The mean cortisol values were $0.082 \mu \mathrm{g} / \mathrm{dL}(S D=0.061)$ at the pretask and $0.090 \mu \mathrm{g} / \mathrm{dL}(S D=0.067)$ at the posttask measurement, with no significant difference between the means at these two time points (paired $t$ test: $t(82)=-$ $1.02, p>0.30$ ). Cortisol change scores, calculated as posttask minus pretask values, ranged from -0.21 to $0.23 \mu \mathrm{g} / \mathrm{dL}$ $(M=0.007, S D=0.062)$.

\section{Performance}

The mean proportion correct was 0.91 ( $S D=0.06$, range 0.74-0.98), and the mean reaction time (RT) was $669 \mathrm{~ms}$ $(S D=132 \mathrm{~ms}$, range $388-1,046 \mathrm{~ms})$. Proportions correct and RTs were inversely correlated $(r=-0.56, p<0.001)$, indicating that participants with higher accuracy tended to respond faster than those with lower accuracy.

\section{ERN and Pe}

Figure 1 illustrates the grand-average response-locked waveforms for correct and error trials. To confirm the presence of the expected ERN in the group as a whole, we conducted an ANOVA on ERN peak amplitudes with Trial Accuracy (error, correct) and Electrode Site (Fz, FCz, Cz, $\mathrm{Pz})$ as repeated measures factors. As expected, the main effect of accuracy reflected greater negative-amplitude peaks for error trials $(M=-6.47 \mu \mathrm{V})$ than for correct trials $(M=-1.24 \mu \mathrm{V})[F(1,82)=142.44, p<0.001]$. Significant effects were also obtained for the main effect of site $[F(3$, $246)=12.93, p<0.001]$ and the Accuracy $\times$ Site interaction $[F(3,246)=35.28, p<0.001]$. As is revealed by the means in Table 1, the effect of trial accuracy on peak amplitudes was greatest at the $\mathrm{FCz}$ site.

Likewise, we conducted an ANOVA on Pe mean amplitudes, including the factors Trial Accuracy and Site. As expected, mean amplitudes were more positive 

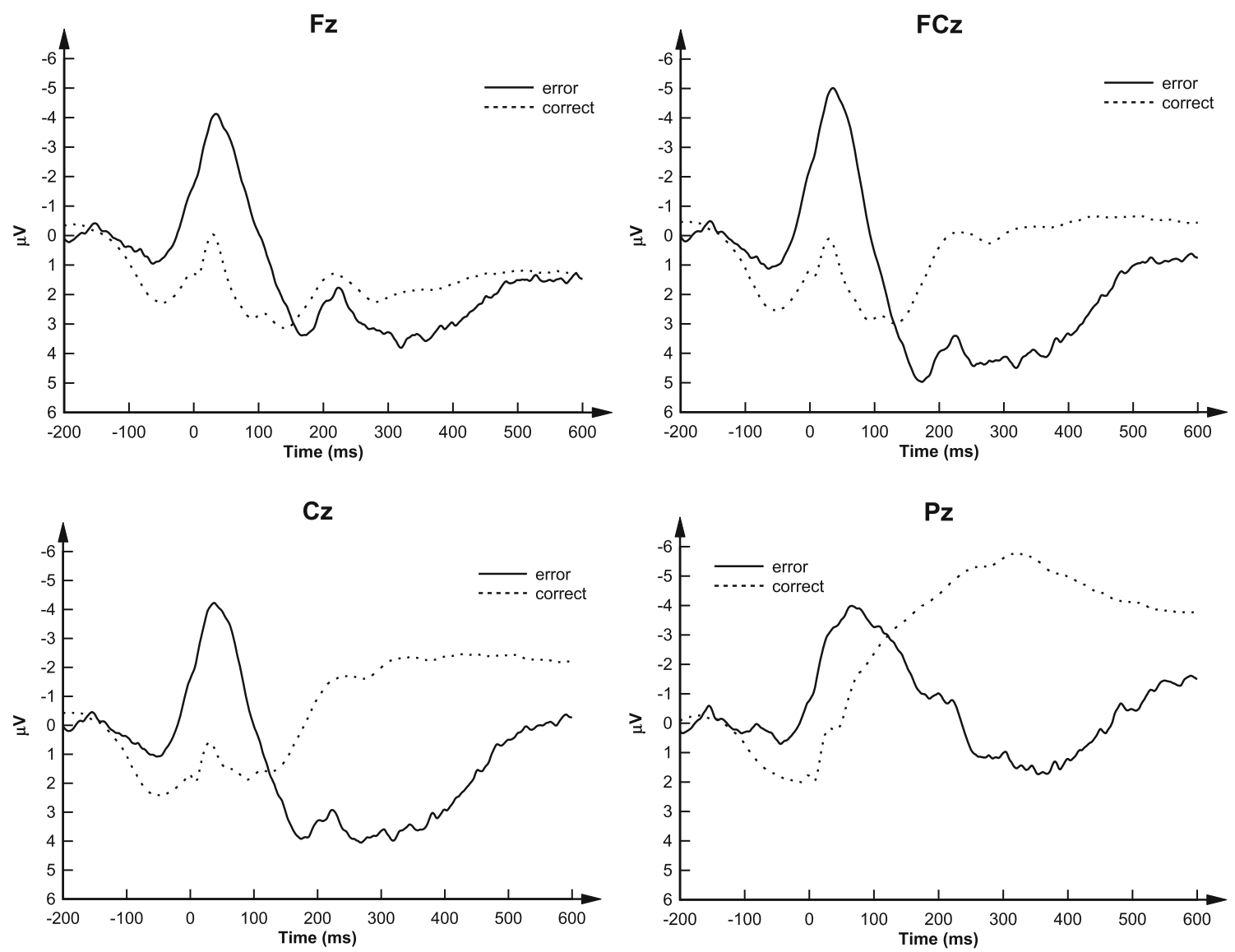

Fig. 1 Grand-average response-locked event-related potentials at the $\mathrm{Fz}, \mathrm{FCz}, \mathrm{Cz}$, and $\mathrm{Pz}$ sites. Time 0 is buttonpress onset

following errors $(M=1.91 \mu \mathrm{V})$ than following correct trials $(M=-1.41 \mu \mathrm{V})[F(1,82)=73.41, p<0.001]$. Means illustrating the main effect of site $[F(3,246)=60.81, p<0.001]$ and the Accuracy $\times$ Site interaction $[F(3,246)=71.29, p<0.001]$ are presented in Table 1. Maximal error-correct differentiation was evident at the $\mathrm{Pz}$ site.

Table 1 Mean (with SEM) amplitudes (in $\mu \mathrm{V}$ ) for correct and error trials in the ERN and Pe time windows

\begin{tabular}{llll}
\hline \multirow{2}{*}{ Electrode Site } & \multicolumn{2}{l}{ Trial Accuracy } & \multirow{2}{*}{ Average } \\
\cline { 2 - 3 } & \multicolumn{1}{l}{ Correct } & Error & \\
\hline ERN (Peak Amplitude $0-100 \mathrm{~ms})$ & & \\
$\mathrm{Fz}$ & $-0.74(0.37)$ & $-6.07(0.46)$ & $-3.41(0.35)$ \\
$\mathrm{FCz}$ & $-0.66(0.39)$ & $-6.97(0.51)$ & $-3.82(0.38)$ \\
$\mathrm{Cz}$ & $-0.59(0.39)$ & $-6.31(0.53)$ & $-3.45(0.40)$ \\
$\mathrm{Pz}$ & $-2.99(0.41)$ & $-6.50(0.51)$ & $-4.75(0.39)$ \\
$\mathrm{Pe}($ Mean Amplitude $200-600 \mathrm{~ms})$ & & \\
$\mathrm{Fz}$ & $1.56(0.51)$ & $2.43(0.51)$ & $2.00(0.47)$ \\
$\mathrm{FCz}$ & $-0.33(0.55)$ & $2.76(0.51)$ & $1.21(0.48)$ \\
$\mathrm{Cz}$ & $-2.14(0.53)$ & $2.21(0.51)$ & $0.32(0.47)$ \\
$\mathrm{Pz}$ & $-4.73(0.51)$ & $0.24(0.49)$ & $-2.24(0.45)$ \\
\hline
\end{tabular}

For purposes of correlating with other variables, we extracted indices reflecting the magnitudes of the ERN and Pe effects for each participant. The ERN index was defined as the difference in the peak amplitudes between error and correct trials at the $\mathrm{FCz}$ site. Values were calculated such that a positive score reflected a greater differentiation between error and correct trials (more-negative amplitudes on error than on correct trials). The mean score on this index was $6.31 \mu \mathrm{V}[S D=4.38$; one-sample $t$ test comparing mean to $0: t(82)=13.11, p<0.001]$. The Pe index was calculated by subtracting the mean amplitude on correct trials from the mean amplitude on error trials, both measured at the $\mathrm{Pz}$ site. The direction of the subtraction meant that more-positive values would indicate greater error-correct differentiation (more-positive amplitudes on error than on correct trials). The mean index score was $2.38 \mu \mathrm{V}[S D=4.22$; one-sample $t$ test: $t(82)=5.14, p<0.001]$.

\section{Alpha suppression}

Log alpha power values were submitted to an ANOVA to confirm the presence of expected patterns of ERAS. The analysis included the repeated measures factors Trial Accuracy (error, correct), Epoch Within the ITI (beginning at the 
time points $0,256,512,768$, and $1,024 \mathrm{~ms}$ after buttonpress), and Electrode Site (including all ten sites, given that prior evidence had shown the effect broadly across the scalp; Carp \& Compton, 2009). All effects in the analysis were significant, as we detail briefly below.

Most importantly, the main effect of accuracy $[F(1,79)=$ $43.29, p<0.001]$ reflected lower alpha power values following errors $\left(M=1.44 \mu \mathrm{V}^{2}\right)$ as compared to correct responses $\left(M=1.50 \mu \mathrm{V}^{2}\right)$, as expected. This effect varied somewhat across the ITI, as was revealed by an Accuracy $\times$ Epoch interaction $[F(4,316)=6.08, p<0.001]$. Figure 2 displays the interaction, as well as illustrating the main effect of epoch $[F(4,316)=13.57, p<0.001]$. Finally, overall alpha power and the magnitude of ERAS varied across sites [main effect of site, $F(9,711)=56.08, p<$ 0.001 ; Accuracy $\times$ Site interaction, $F(9,711)=12.53, p<$ 0.001]. Although ERAS was evident at all sites, it was somewhat more pronounced at parietal sites, consistent with prior findings (Carp \& Compton, 2009; Compton, Arnstein, Freedman, Dainer-Best, \& Liss, 2011); see Table 2 for the means. Finally, the three-way interaction was also significant $[F(36,2844)=5.98, p<0.001]$, although for brevity, means are not presented here; the pattern followed that shown in earlier studies, in which the error-related effect emerged earlier in the epoch at parietal than at other sites.

To simplify analyses relating ERAS to the other variables, we computed a single index that captured the degree of ERAS across all sites and epochs. This index was calculated as the log alpha power following correct trials minus the log alpha power following errors trials, collapsed across epochs and sites. The mean value of this index was $0.064 \mu \mathrm{V}^{2}[S D=$ 0.089 ; one-sample $t$ test comparing mean to $0: t(82)=6.56$, $p<0.001]$. The ERAS index was correlated with the ERN index $(r=0.44, p<0.001)$ but not with the Pe index $(r=-0.08, p>0.45)$, indicating that participants with better

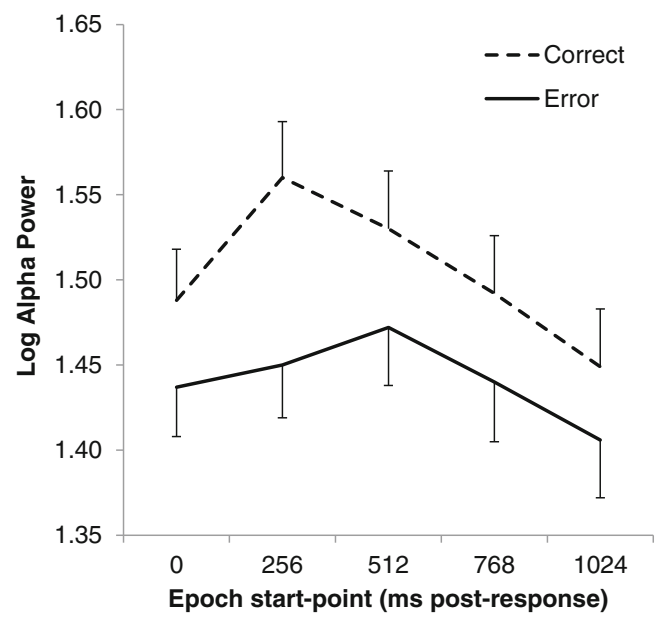

Fig. 2 Log alpha power (in $\mu \mathrm{V}^{2}$ ) during the intertrial interval. Time 0 is the onset of the buttonpress response. Error bars represent SEMs
Table 2 Mean (with $S E M$ ) log alpha power (in $\mu \mathrm{V}^{2}$ ) for correct and error trials across electrode sites

\begin{tabular}{llll}
\hline \multirow{2}{*}{ Electrode Site } & \multicolumn{2}{l}{ Trial Accuracy } & \multirow{2}{*}{ Difference } \\
\cline { 2 - 3 } & Correct & Error & \\
\hline F3 & $1.42(0.03)$ & $1.37(0.03)$ & 0.05 \\
Fz & $1.46(0.03)$ & $1.41(0.03)$ & 0.05 \\
F4 & $1.43(0.03)$ & $1.38(0.03)$ & 0.05 \\
FCz & $1.50(0.03)$ & $1.45(0.03)$ & 0.05 \\
C3 & $1.41(0.03)$ & $1.35(0.03)$ & 0.06 \\
Cz & $1.51(0.03)$ & $1.46(0.03)$ & 0.05 \\
C4 & $1.43(0.03)$ & $1.37(0.03)$ & 0.06 \\
P3 & $1.57(0.04)$ & $1.48(0.04)$ & 0.09 \\
Pz & $1.67(0.04)$ & $1.59(0.04)$ & 0.08 \\
P4 & $1.62(0.04)$ & $1.55(0.04)$ & 0.07 \\
\hline
\end{tabular}

error-correct differentiation in the ERN also tended to show better error-correct differentiation in postresponse alpha power.

Testing for sample differences

Because the data were drawn from two separate samples that completed slightly different versions of the Stroop task, we conducted independent-samples $t$ tests on the cortisol, performance, and EEG/ERP indices to address possible differences between the samples. Means for the two samples are presented in Table 3. The two samples differed in mean RTs $[t(81)=2.19, p<0.05]$, but they did not differ significantly in cortisol change scores, proportions correct, or the ERN, $\mathrm{Pe}$, or ERAS variables.

\section{Relationships among variables}

Table 4 presents zero-order correlations among the cortisol, performance, and ERP/EEG variables. Most notably, the degree of cortisol change was negatively correlated with the ERN index, indicating that participants with larger ERNs showed less cortisol increase during the task (see Fig. 3 for a scatterplot). This relationship remained significant $(p<0.05)$ when the single participant with an ERN score greater than 20 (see Fig. 3) was removed from the analysis, indicating that this individual score was not driving the effect. Neither the Pe nor the ERAS measure predicted cortisol change in the zero-order correlation.

A series of multiple regression analyses were conducted to determine the unique contributions of the error-related neural markers to prediction of cortisol change. The multiple regression approach was selected because two of the error-related neural markers (ERN and ERAS) were intercorrelated with one another, as was reported in previous sections. Furthermore, these variables were intercorrelated 
Table 3 Mean (with $S D$ ) scores for samples 1 and 2

\begin{tabular}{lll}
\hline Variable & \multicolumn{2}{l}{ Sample } \\
\cline { 2 - 3 } & $\begin{array}{l}\text { Sample 1 } \\
(n=47)\end{array}$ & $\begin{array}{l}\text { Sample 2 } \\
(n=36)\end{array}$ \\
\hline Cortisol change & $0.02(0.04)$ & $-0.01(0.08)$ \\
Proportion correct & $0.91(0.06)$ & $0.91(0.06)$ \\
Reaction time $(\mathrm{ms})$ & $696(123)$ & $634(136)$ \\
ERN difference score $(\mu \mathrm{V})$ & $6.04(4.48)$ & $6.66(4.29)$ \\
Pe difference score $(\mu \mathrm{V})$ & $2.59(4.48)$ & $2.10(3.91)$ \\
ERAS difference score $\left(\mu \mathrm{V}^{2}\right)$ & $0.06(0.09)$ & $0.07(0.09)$ \\
\hline
\end{tabular}

with performance variables (see Table 4); generally speaking, greater accuracy and faster RTs were associated with better error-correct differentiation in the ERN and ERAS variables. Therefore, it was necessary to use multiple regression analyses to isolate the unique predictive power of the specific variables.

The first multiple regression analysis focused on the three ERP/EEG variables, predicting cortisol change as a function of the ERN, Pe, and ERAS indices. The full model was significant $\left[F(3,81)=4.94, p<0.005, R^{2}=0.16\right]$. Consistent with the zero-order correlations, the ERN was a unique predictor of cortisol change, $t=-3.28, p<0.005, \beta=-$ 0.383 . In addition, ERAS was a significant predictor in the opposite direction, $t=3.04, p<0.005, \beta=0.348$, meaning that greater ERAS was associated with greater increases in cortisol. The Pe score did not contribute any unique predictive value, $p>0.75$.

An additional regression analysis confirmed that this pattern of predictions held when other possible confounds (mean accuracy, mean RT, sample, gender, and baseline cortisol level) were added simultaneously as predictors in the model. Amongst these nuisance variables, only baseline (pretask) cortisol level significantly predicted unique variance in the cortisol change scores $(t=-3.65, p<0.001, \beta=-$ 0.382 ; higher baseline levels were associated with less cortisol increase during the task). More importantly, the unique variance in cortisol change scores predicted by ERN and ERAS remained robust with all of these nuisance variables controlled [ERN, $t=-2.95, p<0.005, \beta=-0.401$; ERAS,

Table 4 Correlations among cortisol, performance, and EEG measures $(n=83)$

\begin{tabular}{lccc}
\hline & Cortisol Change & Accuracy & Reaction Time \\
\hline Cortisol change & - & -0.05 & 0.19 \\
ERN & $-0.25^{*}$ & $0.54^{* *}$ & $-0.56^{* *}$ \\
Pe & 0.09 & 0.07 & -0.03 \\
Alpha suppression & 0.17 & $0.38^{* *}$ & -0.16 \\
\hline
\end{tabular}

Cell values represent $r$ scores. ${ }^{*} p<0.05$. ${ }^{* *} p<0.001$ $t=3.15, p<0.005, \beta=0.345$; full model, $F(8,81)=4.71$, $\left.R^{2}=0.34, p<0.001\right]$.

A final model was run including a variable representing time of day as a predictor along with the EEG/ERP variables, to make certain that diurnal variations in cortisol could not account for the effects. Because time-of-day information was not available for four of the participants, this analysis was conducted on a sample size of 79 rather than 83 . Nevertheless, the pattern of results remained the same [full model, $F(4,77)=3.59, p=0.01 ; \mathrm{ERN}, t=-3.22, p<0.005$; ERAS, $t=3.04, p<0.005$; effect of time of day, $p>0.40]$.

Following on the regression analyses, we sought to confirm the ERN-related statistical findings with waveform plots that depicted ERNs for participants at different levels of cortisol reactivity. First we divided participants into quartiles based on cortisol change scores (Quartile 1 [Q1], $n=19$, cortisol change scores at or below -0.03 ; Q2, $n=21$, scores -0.02 through $0.00 ; \mathrm{Q} 3, n=23$, scores 0.01 through 0.03; and Q4, $n=20$, scores 0.04 and above). The mean ERN difference scores were 7.78, 5.94, 6.97, and $4.53 \mu \mathrm{V}$ for Quartiles $1-4$, respectively. The Q1 and Q4 means differed significantly (planned contrast, Tukey's LSD, $p<0.05)$ in the direction expected from the scatterplot in Fig. 3 (i.e., larger ERN differences for participants with Q1 cortisol change scores). Q2 and Q3 means were intermediate and not significantly different from the other quartiles. We then plotted waveforms for the Q1 and Q4 groups to confirm the effect visually in the waveforms. As is illustrated in Fig. 4, ERN peaks on error trials were larger for Q1 than for Q4 participants, as expected.

\section{Discussion}

The main finding of this study was that aspects of errorrelated neural signaling are correlated with cortisol regulation, supporting an association between cognitive and emotional self-regulation. Participants whose ERN amplitudes reflected strong differentiation between their own errors and correct responses were less likely to show increased cortisol during the cognitive task, a pattern that remained robust once numerous nuisance variables were controlled. Assuming that cortisol increase is a marker of engagement of the stress response, we inferred that participants with more pronounced ERNs exhibited better stress regulation during the cognitive task.

These findings fit with earlier research demonstrating an association between the ERN and stress reactivity as measured by daily self-reports. In those earlier findings, participants with better error-correct neural differentiation during a Stroop task reported smaller increases in anxiety and more task-focused coping in response to daily stressors (Compton, Arnstein, Freedman, Dainer-Best, Liss, \& Robinson, 2011; Compton et 
Fig. 3 Scatterplot depicting the relationship between ERN (in $\mu \mathrm{V}$ ) and cortisol change (in $\mu \mathrm{g} /$ dL) during the cognitive task. Higher ERN scores reflect better error-correct differentiation in the ERN waveform

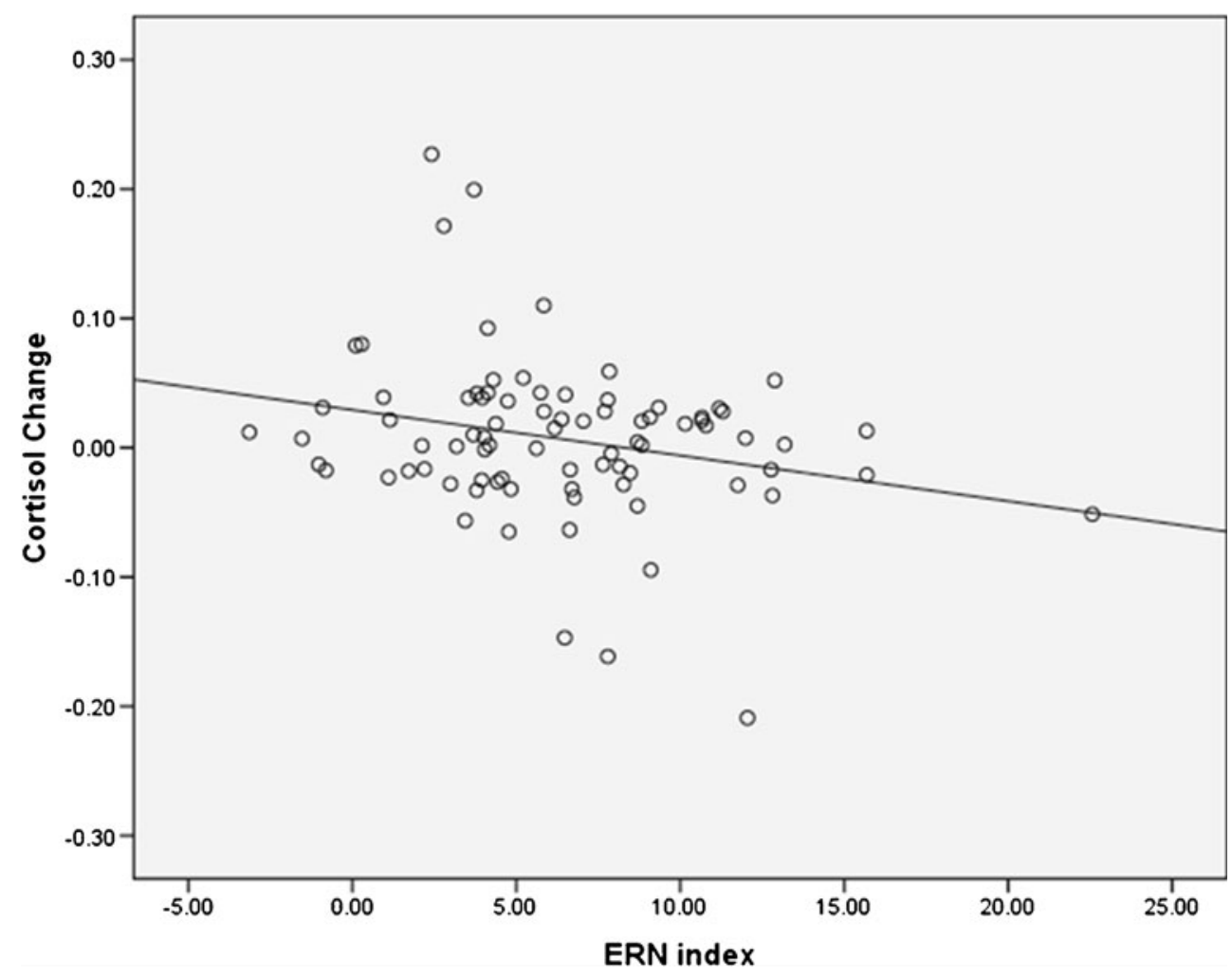

al., 2008). The present results extend these prior findings beyond the self-report assessment of stress regulation, to show that individual differences in the ERN also predict regulation of the stress hormone cortisol.

The most plausible explanation for the association between the ERN and cortisol regulation is the notion that common control processes are crucial to engaging in adaptive performance control and self-regulation of emotion, and that some individuals are more skilled at these processes than others.
This "common systems" view of self-regulation in cognitive and emotional domains fits with other evidence that selfregulation in these domains may draw upon common mental resources (Inzlicht \& Gutsell, 2007; Schmeichel, 2007).

An alternative explanation is that effective cognitive control makes people feel less stressed. That is, participants with greater ERN differentiation may have less of an increase in cortisol during the cognitive task because engaging cognitive control makes the task less stressful, rather than
Fig. 4 Response-locked waveforms at the $\mathrm{FCz}$ site, illustrating ERN peaks for participants in the first (Q1) and fourth (Q4) quartiles of cortisolchange scores

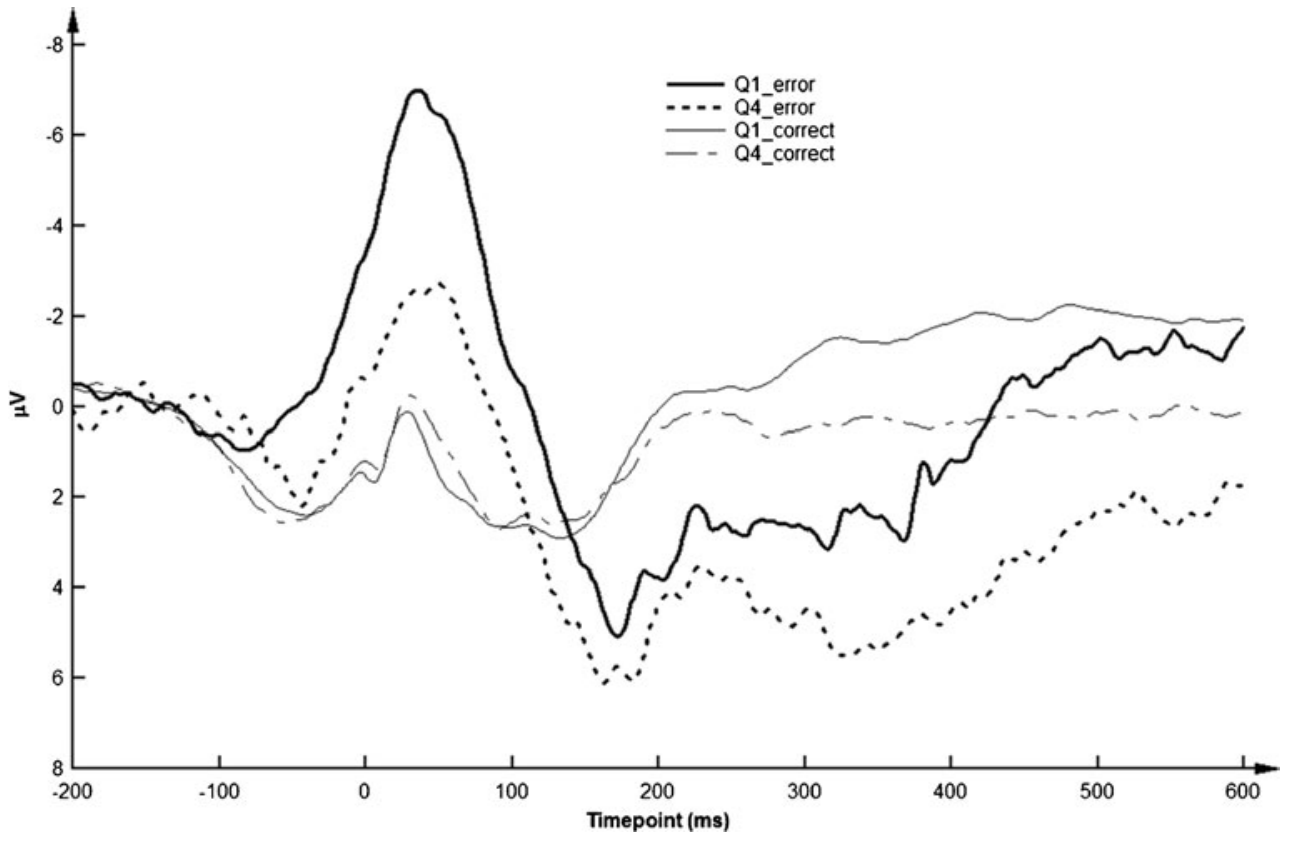


because stress regulation and cognitive control draw upon the same mechanisms. This interpretation, like the first one, speaks to the close relationship between cognitive control and emotion, albeit implicating a slightly different pathway of the causal relationship.

While this second interpretation could be tested further, one aspect of the present results argues against it. Specifically, overall accuracy on the task was not correlated with cortisol change, and the relationship between the ERN and cortisol change remained significant even once the variance shared between the ERN and overall accuracy (and mean RT) was controlled for. If participants are less stressed when cognitive control is better engaged, we would expect to see that relationship mediated by task performance; that is, better task performance ought to be the key factor in making the task feel less stressful. Without that mediating relationship, the alternative explanation must posit that participants feel less stressed when error-related cognitive control is engaged, even if performance isn't improved, a more nuanced explanation that has less intuitive appeal. Nevertheless, future research could attempt to tease apart these two explanations by assessing whether the ERN during a cognitive task predicts cortisol response during an independent stressor (unrelated to the cognitive task itself), as would be predicted by the common-systems explanation.

Another alternative explanation is based on the possibility that cortisol reactivity reflects the mobilization of resources, rather than stress per se (e.g., Tops \& Boksem, 2011a; Tops et al., 2006). Under this conception, the present findings would imply that participants with more efficient error detection, as indexed by the ERN, are also characterized by less mobilization of resources via cortisol. Although somewhat counterintuitive, this explanation could be plausible if participants with greater ERN amplitudes are inherently more absorbed in the task, and therefore have less need to mobilize energy. Indirectly supporting this interpretation, Tops and Boksem (2010) found that individual differences in task absorption are positively correlated with ERN amplitudes. While this alternative interpretation is plausible, it does not have the advantage of also accounting for the prior findings relating ERN and stress reactivity via self-report (e.g., Compton et al., 2008), as does the stress-regulation account of the present findings. Furthermore, to interpret the ERAS data using a similar logic, one would need to assume that those with greater ERAS have less inherent task absorption (and therefore more need to mobilize resources via cortisol), a possibility that would require further empirical validation. Nevertheless, such alternative explanations could be addressed in future studies that quantify task absorption or engagement (e.g., Tops \& Boksem, 2010).

While the present findings fit with a literature indicating that greater error-correct ERN differentiation is beneficial, in the sense of being associated with positive outcome variables, other literature has described clinical conditions, such as obsessive-compulsive disorder, in which the ERN is greater than in controls (for reviews, see Gehring et al., 2012; Olvet \& Hajcak, 2008). Reconciling these two literatures requires abandoning the simplistic notion that a larger ERN is either "better" or "worse" in any universal sense. In addition to the elevated ERNs in some anxiety disorders, reduced ERNs have been observed in clinical conditions such as substance abuse and schizophrenia (Foti, Kotov, Bromet, \& Hajcak, 2012; Gehring et al., 2012; Olvet \& Hajcak, 2008), suggesting that either exaggerated or blunted error responsiveness may characterize clinical conditions. Conceptually, for an adaptive control system to work optimally, an error-monitoring component must detect performance mistakes without triggering "false alarms" that could derail performance. In any case, the present sample focused on individual differences within a nonclinical range, and the findings demonstrate that at least within this sample, greater error sensitivity in the ERN predicted positive outcomes in both performance and cortisol regulation. These findings are consistent with other findings that an enhanced ERN predicts academic achievement (Hirsh \& Inzlicht, 2010) and self-efficacy (Themanson, Pontifex, Hillman, \& McAuley, 2011) within nonclinical samples.

In addition to the main finding associating individual differences in the ERN with cortisol change, we also found that individual differences in ERAS predicted cortisol change. However, this finding only emerged when variance shared with the ERN was partialed out, and interestingly, the pattern of association with cortisol change went in the opposite direction from the pattern involving the ERN. That is, participants with greater alpha suppression following errors showed increased rather than decreased cortisol change scores. In other words, participants who showed greater cerebral arousal following mistakes were more likely to show cortisol increases during the task.

We draw two main conclusions from the alpha suppression results. First, in a general sense these findings support a partial dissociation between the ERN and alpha suppression. Although these two neural indices of error responsiveness have some shared variance, they have different patterns of association with other variables. In prior research, we found that the ERN and ERAS were differently associated with performance variables, such that the ERN predicted posterror accuracy while ERAS predicted posterror slowing (Carp \& Compton, 2009). Here, we found different patterns of association with cortisol change during the task, again supporting the dissociation between the ERN and ERAS.

Furthermore, the alpha suppression findings from the present study, together with other findings, support the interpretation that ERAS is not necessarily an adaptive response to error detection. In recent research, we found that greater ERAS predicts greater levels of anhedonic 
depression, reduced positive affect, and reduced task-focused and affiliative coping behaviors in daily self-reports (Compton, Hofheimer, Kazinka, Levinson, \& Zheutlin, 2012). These findings and those from the present study suggest that ERAS is associated with maladaptive outcomes in the domain of emotion regulation. If alpha suppression reflects generalized cortical arousal, participants with higher posterror arousal appear to regulate stress more poorly than do those with lower posterror arousal. Posterror arousal may also have negative consequences for cognitive performance, as suggested by our previous findings that greater ERAS predicts greater posterror slowing without any benefit to posterror accuracy (Carp \& Compton, 2009).

While the ERN and ERAS emerged as significant independent predictors of the stress response, albeit in opposite directions, the Pe did not emerge as a significant predictor of cortisol change either in the zero-order correlations or in any of the multiple regression analyses. These results are somewhat at odds with the interpretation that the Pe is closely tied to the emotional response to errors, as was initially proposed by researchers who found that the Pe amplitude was associated with autonomic activity following errors (Hajcak et al., 2003). However, before discarding that interpretation, key differences between that prior study and the present one should be noted. In the prior study, Hajcak et al. examined autonomic variables that could vary on a trial-to-trial basis, namely heart rate and skin conductance; in contrast, cortisol, a hormonal variable with a more sluggish response, could not be expected to vary on a trial-to-trial basis. As a result, the prior study was able to measure autonomic responses to errors themselves, whereas the present study measured cortisol responses to the entire task. Therefore, it remains possible (although somewhat counterintuitive) that the process reflected by the Pe is one that engages autonomic responses transiently in response to the occurrence of errors but is not related to the overall experience of stress, arousal, or engagement during the task session.

Alternatively, the present results may fit better with the theory that the Pe is not specifically tied to mechanisms of emotional arousal in response to errors, but rather reflects a cognitive response to rare events. Researchers have distinguished between an "early Pe," an error-related positivity that has a frontocentral distribution and follows the ERN rather immediately, and a "late Pe" that is more sustained and has a posterior scalp distribution (Arbel \& Donchin, 2009). The Pe as measured in the present study was analogous to the late $\mathrm{Pe}$, given that it was sustained during 200$600 \mathrm{~ms}$ following the response, with error-correct differentiation being maximal at parietal sites. Due to the scalp distribution and polarity of the late $\mathrm{Pe}$, researchers have proposed that it may reflect a P3 oddball response, with the "oddball" being an error, which occurs rarely among more-frequent correct responses (e.g., Arbel \& Donchin,
2009; Ridderinkhof et al., 2009). Such an interpretation of the Pe would not necessarily predict an association between the Pe and emotion regulation, and therefore could be consistent with the null findings in the present study (but see Compton, Arnstein, Freedman, Dainer-Best, Liss, \& Robinson, 2011, for associations between Pe and self-reported measures of emotion regulation).

A strength of the present study is the combination of ERP/EEG measures of cognitive control and cortisol measures of stress regulation in a sample size large enough for relatively modest associations to be detected statistically. Although the effect sizes for the ERN and ERAS as predictors of cortisol change were modest in magnitude, they remained statistically significant once controlling for numerous nuisance variables, suggesting a reliable finding. Indeed, the fact that ERN and alpha suppression measures continued to predict cortisol response even after controlling for individual differences in task performance indicates the potential of these EEG measures to yield clues about individual differences in self-regulation that could not be gleaned from behavioral performance measures alone.

At the same time, the study is not without limitations. Most notably, the fact that cortisol reactivity was measured during the cognitive task may have maximized the possibility of association between the cognitive control and cortisol variables. In addition, the group as a whole did not exhibit significant changes in mean cortisol levels from the pretask to posttask measurements, suggesting that the Stroop task was not consistently perceived as a stressor. Indeed, cortisol changes appear to be largest when the stressor involves some aspect of social-evaluative threat (Dickerson \& Kemeny, 2004), which may not have been consistently perceived in the present task. This latter point is only a weak limitation, because individual differences can be informative even when the group average is not. Indeed, the goal of the study was to examine individual differences in the cortisol response during the task, not to demonstrate that the task was stressful for all (or even most) participants.

Even stronger evidence for the concept of overlapping mechanisms contributing to cognitive and emotional selfregulation could come from future studies that examine whether individual differences in error-related cognitive control predict cortisol reactivity to other kinds of stressors, such as more standard laboratory stress induction paradigms or daily-life stressors. Our prior findings indicated that error-related cognitive control predicts aspects of stress regulation as measured by self-reports over a period of two weeks subsequent to the cognitive task (Compton, Arnstein, Freedman, Dainer-Best, Liss, \& Robinson, 2011; Compton et al., 2008), so there is reason to predict that cortisol reactivity might also be predicted by cognitive control measures taken under independent conditions; nevertheless, this remains an empirical question for future research. 
Author note This research was supported by NIH Grant No. R15MH085182 to R.J.C. Assistance in the data collection was provided by Daniel Arnstein, Justin Dainer-Best, Gili Freedman, Amanda Levinson, Alison Liss, and Amanda Zheutlin.

\section{References}

Amen, A., Dodd, J., Fineman, S. L., \& Compton, R. J. (2008). Individual differences in cognitive control are associated with cortisol reactivity. Psychophysiology, 45(Suppl. 1), S49.

Arbel, Y., \& Donchin, E. (2009). Parsing the componential structure of post-error ERPs: A principal components analysis of ERPs following errors. Psychophysiology, 46, 1179-1189. doi:10.1111/ j.1469-8986.2009.00857.x

Barbas, H., Saha, S., Rempel-Clower, N., \& Ghashghaei, T. (2003). Serial pathways from primate prefrontal cortex to autonomic areas may influence emotional expression. BMC Neuroscience, 4, 25. doi:10.1186/1471-2202-4-25

Barsegyan, A., McKenzie, S. M., Kurose, B. D., McGaugh, J. L., \& Roozendaal, B. (2010). Glucocorticoids in the prefrontal cortex enhance memory consolidation and impair working memory by a common neural mechanism. Proceedings of the National Academy of Sciences, 107, 16655-16660. doi:10.1073/pnas.1011975107

Baumeister, R. F., \& Vohs, K. D. (2004). Handbook of self-regulation: Research, theory, and practice. New York: Guilford Press.

Botvinick, M. M., Braver, T. S., Barch, D. M., Carter, C. S., \& Cohen, J. D. (2001). Conflict monitoring and cognitive control. Psychological Review, 108, 624-652. doi:10.1037/0033-295X.108.3.624

Carp, J., \& Compton, R. J. (2009). Alpha power is influenced by performance errors. Psychophysiology, 46, 336-343. doi:10.1111/j.1469-8986.2008.00773.x

Cavanagh, J. F., \& Allen, J. J. B. (2008). Multiple aspects of the stress response under social evaluative threat: An electrophysiological investigation. Psychoneuroendocrinology, 33, 41-53. doi:10.1016/ j.psyneuen.2007.09.007

Compton, R. J., Arnstein, D., Freedman, G., Dainer-Best, J., \& Liss, A. (2011). Cognitive control in the inter-trial interval: Evidence from EEG alpha power. Psychophysiology, 48, 583-590. doi:10.1111/ j.1469-8986.2010.01124.x

Compton, R. J., Arnstein, D., Freedman, G., Dainer-Best, J., Liss, A., \& Robinson, M. D. (2011). Neural and behavioral measures of error-related cognitive control predict daily coping with stress. Emotion, 11, 379-390. doi:10.1037/a0021776

Compton, R. J., Hofheimer, J., Kazinka, R., Levinson, A. R., \& Zheutlin, A. (2012). Alpha suppression following performance errors is correlated with depression, affect and coping behaviors. Manuscript under review.

Compton, R. J., Robinson, M. D., Ode, S., Quandt, L. C., Fineman, S. L., \& Carp, J. (2008). Error-monitoring ability predicts daily stress regulation. Psychological Science, 19, 702-708. doi:10.1111/j.1467-9280.2008.02145.x

Dickerson, S. S., \& Kemeny, M. E. (2004). Acute stressors and cortisol responses: A theoretical integration and synthesis of laboratory research. Psychological Bulletin, 130, 355-391. doi:10.1037/ 0033-2909.130.3.355

Falkenstein, M., Hohnsbein, J., Hoormann, J., \& Blanke, L. (1991). Effects of cross-modal divided attention on late ERP components: II. Error processing in choice reaction tasks. Electroencephalography and Clinical Neurophysiology, 78, 447-455. doi:10.1016/ 0013-4694(91)90062-9

Foti, D., Kotov, R., Bromet, E., \& Hajcak, G. (2012). Beyond the broken error-related negativity: Functional and diagnostic correlates of error processing in psychosis. Biological Psychiatry, 71, 864-872. doi:10.1016/j.biopsych.2012.01.007

Gehring, W. J., Goss, B., Coles, M. G. H., Meyer, D. E., \& Donchin, E. (1993). A neural system for error detection and compensation. Psychological Science, 4, 385-390. doi:10.1111/ j.1467-9280.1993.tb00586.x

Gehring, W. J., Liu, Y., Orr, J. M., \& Carp, J. (2012). The error-related negativity (ERN/Ne). In S. J. Luck \& E. S. Kappenman (Eds.), The oxford handbook of event-related potentials (pp. 231-291). New York: Oxford.

Gross, J. J. (2007). Handbook of emotion regulation. New York: Guilford Press.

Hajcak, G., McDonald, N., \& Simons, R. F. (2003). To err is autonomic: Error-related brain potentials, ANS activity, and post-error compensatory behavior. Psychophysiology, 40, 895-903. doi:10.1111/1469-8986.00107

Hirsh, J. B., \& Inzlicht, M. (2010). Error-related negativity predicts academic performance. Psychophysiology, 47, 192-196. doi:10.1111/j.1469-8986.2009.00877.x

Hofmann, W., Schmeichel, B. J., \& Baddeley, A. D. (2012). Executive functions and self-regulation. Trends in Cognitive Sciences, 16, 174-180. doi:10.1016/j.tics.2012.01.006

Holroyd, C. B., \& Coles, M. G. H. (2002). The neural basis of human error processing: Reinforcement learning, dopamine, and the error-related negativity. Psychological Review, 109, 679-709. doi:10.1037/0033-295X.109.4.679

Inzlicht, M., \& Gutsell, J. N. (2007). Running on empty: Neural signals for self-control failure. Psychological Science, 18, 933-937. doi:10.1111/j.1467-9280.2007.02004.x

MacKenzie, M. B., Mezo, P. G., \& Francis, S. E. (2012). A conceptual framework for understanding self-regulation in adults. New Ideas in Psychology, 30, 155-165. doi:10.1016/j.newideapsych.2011.07.001

Ochsner, K. N., \& Gross, J. J. (2005). The cognitive control of emotion. Trends in Cognitive Sciences, 9, 242-249. doi:10.1016/j.tics.2005.03.010

Olvet, D. M., \& Hajcak, G. (2008). The error-related negativity (ERN) and psychopathology: Toward an endophenotype. Clinical Psychology Review, 28, 1343-1354. doi:10.1016/ j.cpr.2008.07.003

Overbeek, T. M., Nieuwenhuis, S., \& Ridderinkhof, K. R. (2005). Dissociable components of error processing: On the functional significance of the Pe vis-à-vis the ERN/Ne. Journal of Psychophysiology, 19, 319-329. doi:10.1027/0269-8803.19.4.319

Ray, R. D., \& Zald, D. H. (2012). Anatomical insights into the interaction of emotion and cognition in the prefrontal cortex. Neuroscience and Biobehavioral Reviews, 36, 479-501. doi:10.1016/j.neubiorev.2011.08.005

Ridderinkhof, K. R., Ramautar, J. R., \& Wijnen, J. G. (2009). To Pe or not to Pe: A P3-like ERP component reflecting the processing of response errors. Psychophysiology, 46, 531-538. doi:10.1111/ j.1469-8986.2009.00790.x

Ridderinkhof, K. R., van den Wildenberg, W. P. M., Segalowitz, S. J., \& Carter, C. S. (2004). Neurocognitive mechanisms of cognitive control: The role of prefrontal cortex in action selection, response inhibition, performance monitoring, and reward-based learning. Brain and Cognition, 56, 129-140. doi:10.1016/j.bandc.2004.09.016

Roozendaal, B., McReynolds, J. R., Van der Zee, E. A., Lee, S., McGaugh, J. L., \& McIntyre, C. K. (2009). Glucocorticoid effects on memory consolidation depend on functional interactions between the medial prefrontal cortex and basolateral amygdala. Journal of Neuroscience, 29, 14299-14308. doi:10.1523/JNEUROSCI.3626-09.2009

Schmeichel, B. J. (2007). Attention control, memory updating, and emotion regulation temporarily reduce the capacity for executive 
control. Journal of Experimental Psychology. General, 136, 241255. doi:10.1037/0096-3445.136.2.241

Simons, R. F. (2010). The way of our errors: Theme and variations. Psychophysiology, 47, 1-14. doi:10.1111/j.1469-8986.2009.00929.x

Themanson, J. R., Pontifex, M. B., Hillman, C. H., \& McAuley, E. (2011). The relation of self-efficacy and error-related selfregulation. International Journal of Psychophysiology, 80, 1-10. doi:10.1016/j.ijpsycho.2011.01.005

Tops, M., \& Boksem, M. A. S. (2010). Absorbed in the task: Personality measures predict engagement during task performance as tracked by error negativity and asymmetrical frontal activity. Cognitive, Affective, \& Behavioral Neuroscience, 10, 441-453. doi:10.3758/CABN.10.4.441

Tops, M., \& Boksem, M. A. S. (2011a). Cortisol involvement in mechanisms of behavioral inhibition. Psychophysiology, 48, 723-732. doi:0.1111/j.1469-8986.2010.01131.x

Tops, M., \& Boksem, M. A. S. (2011b). A potential role for the inferior frontal gyrus and anterior insula in cognitive control, brain rhythms, and event-related potentials. Frontiers in Psychology, 2 (330), 1-14. doi:10.3389/fpsyg.2011.00330

Tops, M., Boksem, M. A. S., Wester, A. E., Lorist, M. M., \& Meijman, T. F. (2006). Task engagement and the relationships between the error-related negativity, agreeableness, behavioral shame proneness and cortisol. Psychoneuroendocrinology, 31, 847-858. doi:10.1016/j.psyneuen.2006.04.001

van Veen, V., \& Carter, C. S. (2006). Conflict and cognitive control in the brain. Current Directions in Psychological Science, 15, 237240. doi:10.1111/j.1467-8721.2006.00443.x

Veer, I. M., Oei, N. Y. L., Spinhoven, P., van Buchem, M. A., Elzinga, B. M., \& Ronbouts, S. A. R. B. (2012). Endogenous cortisol is associated with functional connectivity between the amygdala and medial prefrontal cortex. Psychoneuroendocrinology, 37, 10391047. doi:10.1016/j.psyneuen.2011.12.001

Zelazo, P. D., \& Cunningham, W. A. (2007). Executive function: Mechanisms underlying emotion regulation. In J. J. Gross (Ed.), Handbook of emotion regulation (pp. 135-158). New York: Guilford Press. 